\title{
INFLUENCING THE AERODYNAMICS OF THE ACFA2020 AIRCRAFT WITH FLAP AND TRAILING EDGE DEVICE OSCILLATIONS
}

\section{Meyer ${ }^{1}$ and Ch. Breitsamter ${ }^{2}$}

\author{
${ }^{1}$ Technische Universität München \\ Institute of Aerodynamics and Fluidmechanics \\ 15 Boltzmannstraße, Garching 85748, Germany \\ (now at EADS Innovation Works) \\ ${ }^{2}$ Technische Universität München \\ Institute of Aerodynamics and Fluidmechanics \\ 15 Boltzmannstraße, Garching 85748, Germany
}

\begin{abstract}
The influence of an oscillating aileron and trailing edge device on the unsteady aerodynamics of a blended wing body (BWB) aircraft configuration with high-fidelity time-accurate Euler simulations has been investigated. Steady results show an unequally-distributed lift distribution in spanwise direction with a particularly severe shock at cruise conditions on the outboard wing. Unsteady oscillations of the outboardlocated aileron are able to influence the local and global aerodynamics. The oscillation of the trailing edge device designed to be at trailing edge of the aileron does not show any great effect on neither local nor global aerodynamics.
\end{abstract}

\section{INTRODUCTION}

The ACFA2020 (Active Control for Flexible 2020 Aircraft) is the European vision of an ultraefficient, marketable BWB aircraft for the year 2020. Innovative designs such as ACFA2020 are needed to fulfill the ambitious goals of the Advisory Council for Aeronautics Research in Europe (ACARE) to reduce the fuel consumption, $\mathrm{CO}_{2}$-emissions by $50 \%$, and the external noise by $4-5 \mathrm{~dB}$.

Previous flying wing projects, e. g., VELA (Very Efficient Large Aircraft) and NACRE (New Aircraft Concepts Research), have identified a high need for active 
control and related expertise [1]. The innovation of active control is, therefore, the major focus of the ACFA2020 project [2]. Since flight control design and flight performance are strongly influenced by the aircraft aerodynamics, accurate aerodynamic predictions are required even in the preliminary design process. Despite the strong nonlinear phenomena occurring on the BWB results of acceptable accuracy can only be obtained by high-fidelity simulations as opposed to simplified approaches, e. g., empirical methods [3].

Within the ACFA2020 project, high-fidelity steady and unsteady aerodynamic simulations were employed in order to provide aerodynamic input data for setting up reduced order models used in the control system design. The unsteady input data were delivered as generalized aerodynamics forces which serve as excitation forces used in the design of active control of flexible aircraft modes.

The objective of this work is to analyze the steady high-speed aerodynamics and the unsteady aerodynamics due to oscillating control surfaces, e. g., flap and trailing edge devices. Control surfaces can be used to alleviate aerodynamic loads in order to reduce structural strain. This can lead to a reduction in mass and, respectively, operational cost.

Above all, the aim is to answer the question whether it is sufficient to use a small trailing edge device instead of the aileron to generate excitation forces of the required magnitude.

\section{NUMERICAL METHOD}

In this study, the solver AER-Eu is employed which uses an Euler set of equations discretized on structured, collocated finite-volume grids [4]. The strongly conservative form guarantees shock-capturing without any additional effort. A Total Variation Diminishing (TVD) scheme prevents unphysical oscillations [5]. The numerical convective fluxes are computed by the Roe scheme [6]. For time advancement, the current calculations use a time-accurate scheme with LowerUpper Symmetric Successive OverRelaxation (LU-SSOR) [7]. The numerical solver is second-order accurate in time and space.

One special feature of AER-Eu is the capability to simulate flows about deforming/deplacing geometries. An example for a deformed geometry is an elastic mode shape like symmetric bending or a control surface oscillation. An example for a displaced geometry is a plunge movement. For calculations with deformed/displaced geometries, both displaced/deformed and undisplaced/ undeformed spatial grids are required. As initial solution, a steady-state solution of the undisplaced/undeformed aircraft is used. The data format of the results guarantees a direct use in commonly applied aeroelastic analysis tools, e. g, Nastran or ZAERO. 
In ACFA2020, the aerodynamic data is input to a coupled iterative flightdynamics-analysis structure-sizing process. The flight-load-analysis tool uses the aerodynamic data to deliver critical airframe loads which are needed by the structure calculation tool for optimization.

\section{STUDY}

\subsection{Test Case Description}

The ACFA2020 BWB aircraft has a root chord length $\left(c_{r}\right)$ of $43 \mathrm{~m}$, a Mean Aerodynamic Chord (MAC) of $25 \mathrm{~m}$, a half-spanwidth $(b)$ of $40 \mathrm{~m}$, and a reference area $\left(S_{\text {ref }}\right)$ of $1323 \mathrm{~m}^{2}$ (Fig. 1). The basic configuration avails of large winglets with a rudder for lateral control and five large flaps for longitudinal control. For studying the influence of oscillating control surfaces on the aerodynamics, Flap5/aileron and a Trailing Edge Device (TED) are selected. The maximum deflection angle for both Flap5 and TED is about $2^{\circ}$.

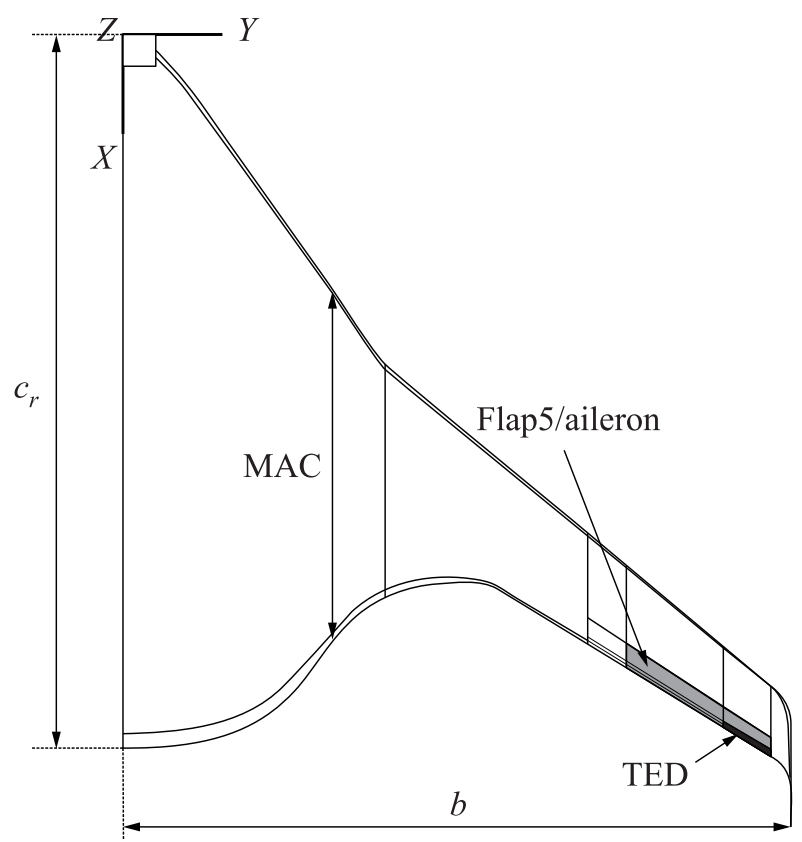

Figure 1 Overview on ACFA2020 with Flap5/aileron and TED 


\subsection{Conventions}

The Angle of Attack (AoA) is given in degrees with respect to the $x z$-plane of the coordinate system shown in Fig. 1. For the oscillations the reduced frequency is

$$
k_{\text {red }}=\frac{\omega \cdot \mathrm{MAC}}{2 u}
$$

where $u$ is the flight speed and $\omega$ is the angular frequency of the unsteady motion. Pressure distribution plots will be given at mid-flap positions in a local streamwise coordinate system which is defined to be $\left(x_{\mathrm{le}}-x\right) / c$ where $x_{\mathrm{le}}$ is the position of the airfoil's leading edge and $c$ is its local chord length. The pressure coefficient used in the analysis is expressed by

$$
C_{p}=\frac{p-p_{\infty}}{0.5 \rho_{\infty} u_{\infty}^{2}}
$$

where $p$ is the local pressure; and $p_{\infty}$ and $\rho_{\infty}$ are the ambient pressure and the density, respectively.

For the analysis of the Flap5 and TED oscillations, the pressure coefficient's evolutions of the time-accurate simulations undergo a posteriori spectral analysis. The resulting real and imaginary part of the pressure coefficient distribution serve as input in the calculation of unsteady aerodynamic forces, i. e., Generalized Aerodynamics Forces (GAFs). How the GAFs are used as excitation forces in the reduced order models of the control system design is explained briefly in the following. The time-dependent displacement vector $\mathbf{X}(t)$, i. e., deployment of Flap5 or TED, leads to the external (aerodynamic) force $\mathbf{F}_{\text {aer }}(\mathbf{X}(t)$ ) in the classical dynamic equation

$$
\mathbf{M} \ddot{\mathbf{X}}+\mathbf{K} \dot{\mathbf{X}}=\mathbf{F}_{\text {aer }}(\mathbf{X}(t))
$$

where $\mathbf{M}$ is the mass matrix and $\mathbf{K}$ is the stiffness matrix. Structural damping is neglected here. With the modal matrix $\boldsymbol{\Phi}$ and the vector of the modal amplitudes q, the modal transformation

$$
\mathbf{X}(p)=\mathbf{\Phi} \cdot \mathbf{q}(p)
$$

can be defined. Here, $p$ is the dimensionless Laplace-domain variable. With the modal transformation and some mathematical operations, Eq. (1) can be transformed from the time-domain into the frequency-domain:

$$
\left(p \frac{\mathrm{MAC}}{u_{\infty}}\right)^{2} \mathbf{M}_{\mathrm{gen}} \mathbf{q}(p)+\mathbf{K}_{\mathrm{gen}} \cdot \mathbf{q}(p)=\boldsymbol{\Phi}^{\mathrm{T}} \mathbf{F}_{\mathrm{aer}}(p)
$$


where $\mathbf{M}_{\text {gen }}=\boldsymbol{\Phi}^{\mathrm{T}} \mathbf{M} \boldsymbol{\Phi}$ and $\mathbf{K}_{\text {gen }}=\boldsymbol{\Phi}^{\mathrm{T}} \mathbf{K} \boldsymbol{\Phi}$ are the generalized mass- and stiffness-matrix. Since here the aerodynamic forces are the solution of a linearized differential equation in time, they can be written in the Laplace-domain as product of a transfer function matrix with the displacements $\mathbf{X}$ in the physical basis, or with the modal amplitudes $\mathbf{q}$ in the modal space. Then, Eq. (2) becomes

$$
\left(p \frac{\mathrm{MAC}}{u_{\infty}}\right)^{2} \mathbf{M}_{\mathrm{gen}} \cdot \mathbf{q}(p)+\mathbf{K}_{\mathrm{gen}} \cdot \mathbf{q}(p)=q_{\infty} \mathbf{G A F} \cdot \mathbf{q}(p)
$$

where $\mathbf{G A F}$ is the matrix for the generalized aerodynamic forces.

\subsection{Numerical Setup}

For all simulations, a grid with a favorable OO-block topology is used. The domain extents are chosen such that far-field boundary conditions do not influence the flow physics near the aircraft. The grid consists of 1 million computational cells. Grid independency was proven by a grid convergence study. All grids have been generated in the commercial tool ANSYS ICEM-CFD. For better convergence property and accuracy, all grids were optimized with an in-house globally-elliptic smoothing tool.

Table 1 Investigated flow cases with ranges for flight Mach number (M), AoA, and reduced frequency $\left(k_{\text {red }}\right)$

\begin{tabular}{cccc}
\hline Case & $\mathrm{M}$ & AoA & $k_{\text {red }}$ \\
\hline Steady & $0.75,0.85,0.88$ & $1.7^{\circ}$ & - \\
Steady & 0.85 & $0.4^{\circ}, 1.7^{\circ}, 3.0^{\circ}$ & - \\
Oscillating Flap5 & $0.75,0.85$ & $1.7^{\circ}$ & $0.01,0.05,0.1,0.5,1.0,2.0$ \\
Oscillating TED & $0.75,0.85$ & $1.7^{\circ}$ & $0.01,0.05,0.1,0.5,1.0,2.0$ \\
\hline
\end{tabular}

\subsection{Results}

The focus is on the test cases summarized in Table 1. In addition to the steady simulation cases, unsteady simulations with oscillating control surfaces (Flap5 and TED (see Fig. 1)) are used to investigate the influence on the BWB aerodynamics.

\subsubsection{Steady results}

Aerodynamics on the BWB are determined by strong nonlinear effects. A complex shock system is built up on the upper and lower parts of the wing and the winglet for cruise conditions $\left(\mathrm{M}=0.85\right.$ and $\left.\mathrm{AoA}=1.7^{\circ}\right)$ shown in Fig. 2. 


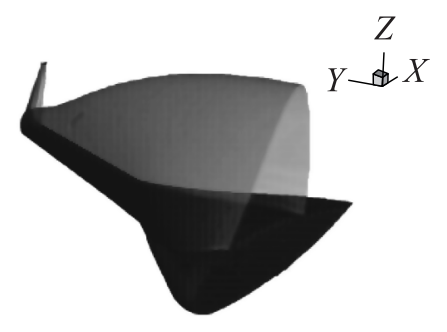

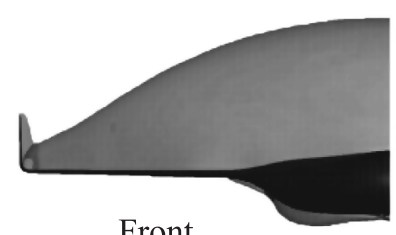

Front
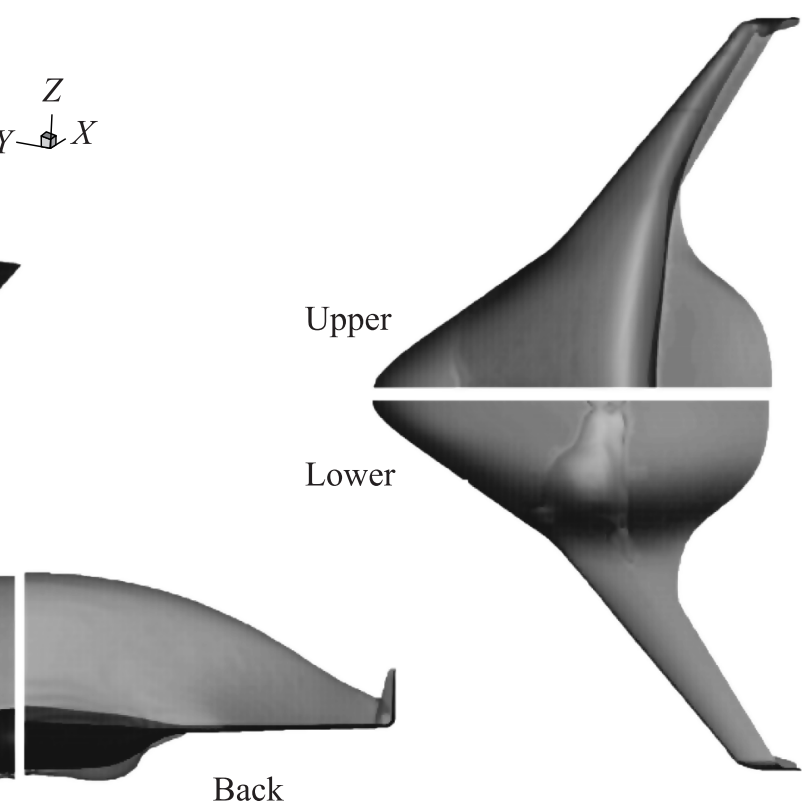

Back

Figure 2 Mach = 1-isosurface at the front, back, upper, and lower parts of the BWB at $\mathrm{M}=0.85$ and $\mathrm{AoA}=1.7^{\circ}$

With increasing Mach number, the shock system moves towards the trailing edge of the wing which is visible in the pressure coefficient plots in Fig. 3. The shock positions differ strongly in spanwise direction. On the outboard wing, the shock position variation is severe. This variation of the shock position implies an undesirable unequally distributed wing lift distribution leaving potential for performance improvement.

\subsubsection{Unsteady results}

The influence of an oscillating Flap5/aileron or TED on the unsteady aerodynamics has been investigated at $\mathrm{AoA}=1.7^{\circ}$ and $\mathrm{M}=0.85$ and 0.75 . Oscillation is performed at the frequencies summarized in Table 1.

Figures 4 and 5 show the real and the imaginary part of $C_{p}$ for the previouslyused outboard mid-flap position for the case of an oscillating Flap5/aileron. The pressure peaks indicate a shock position. The amplitude of the peak corresponds to the shock's strength and the width of the peak to the range of the shock movement due to the oscillation. 

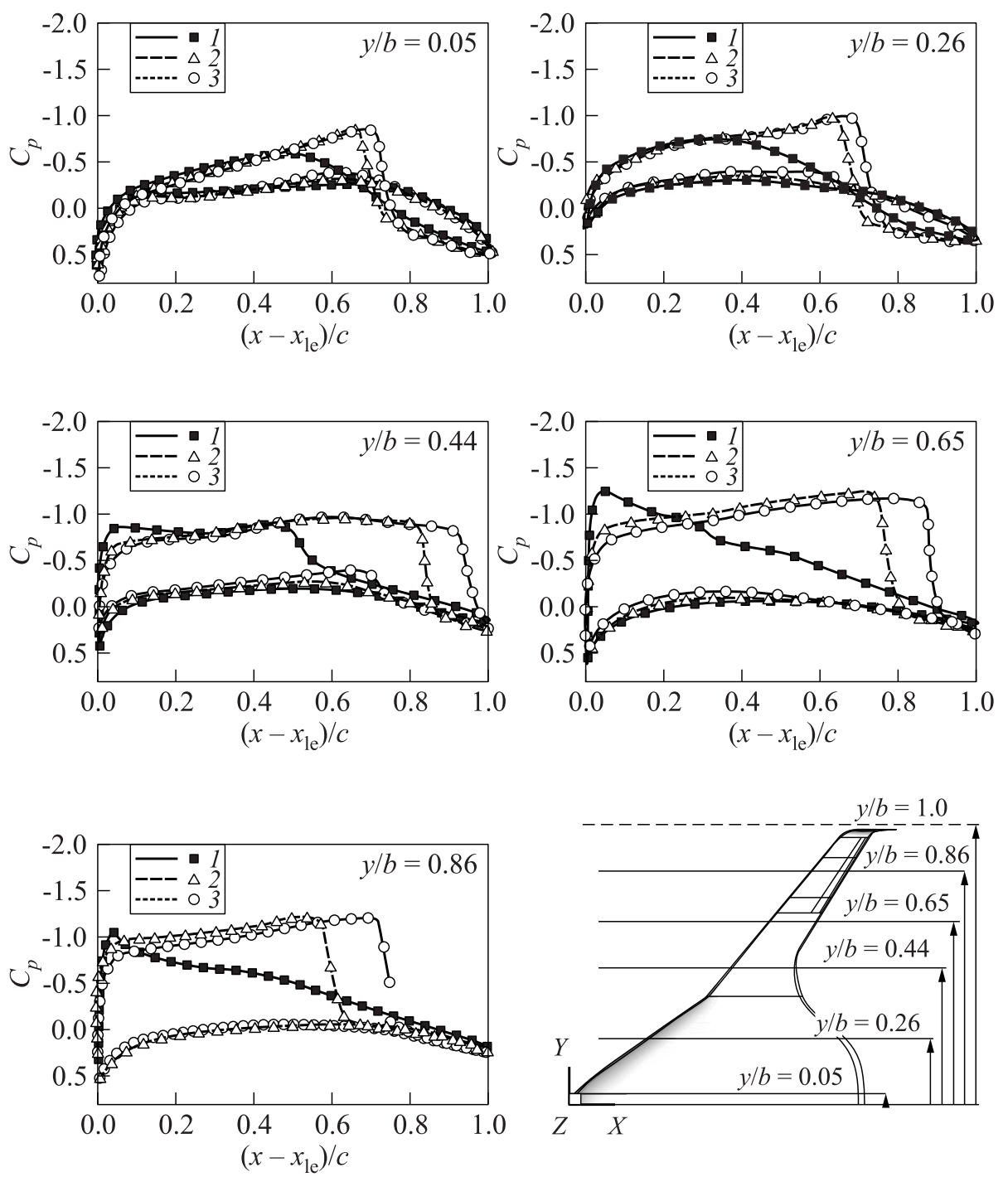

Figure 3 The pressure coefficient in streamwise direction $\left(x-x_{\mathrm{le}}\right) / c$ at different spanwise positions $y / b$ for different Mach numbers $(1-\mathrm{M}=0.75 ; 2-0.85$; and $3-$ $\mathrm{M}=0.88)$ and $\mathrm{AoA}=1.7^{\circ}$ 

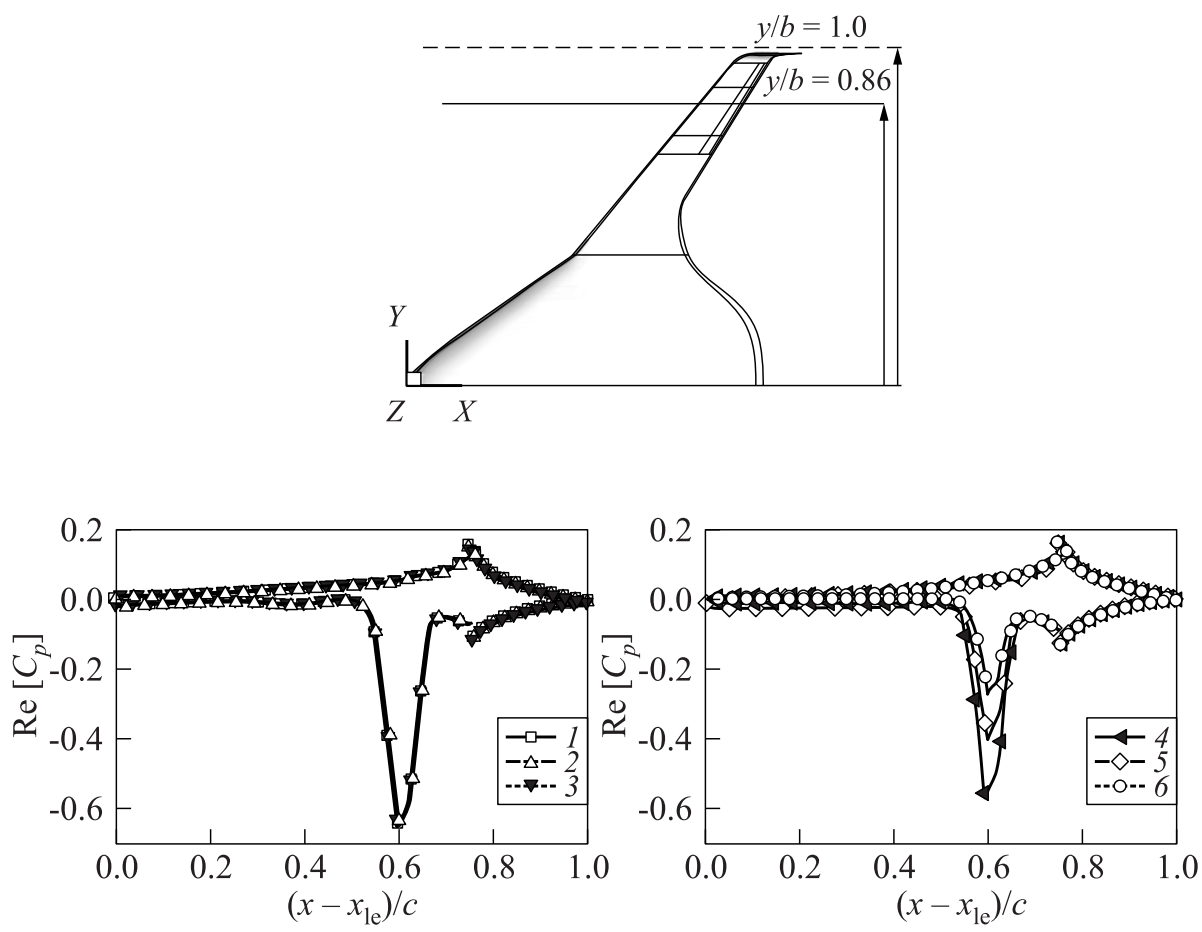

(a)
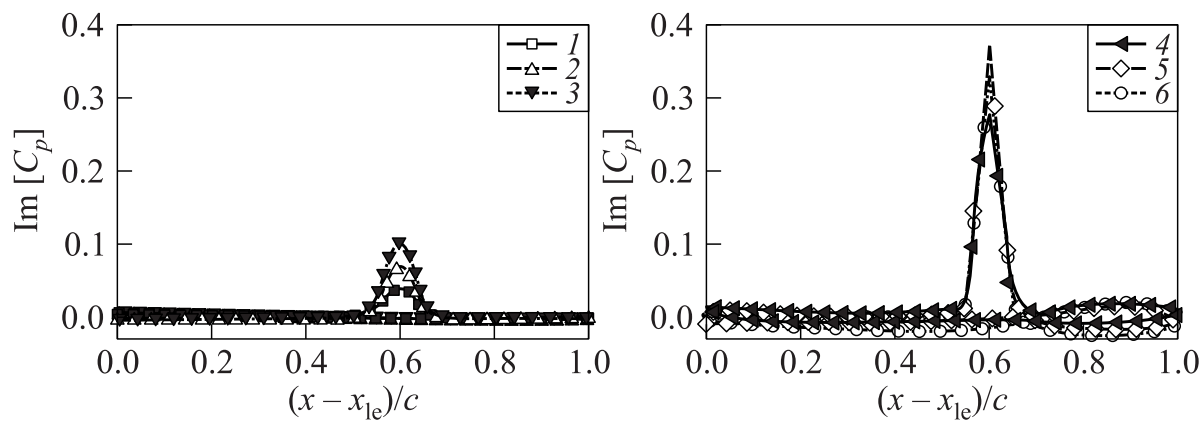

$(b)$

Figure 4 Real $(a)$ and imaginary $(b)$ parts of the pressure coefficient for oscillating Flap5, $\mathrm{M}=0.85, y / b=0.86: 1-k_{\text {red }}=0.01 ; 2-0.05 ; 3-0.1 ; 4-0.5 ; 5-1.0$; and $6-k_{\text {red }}=2.0$ 

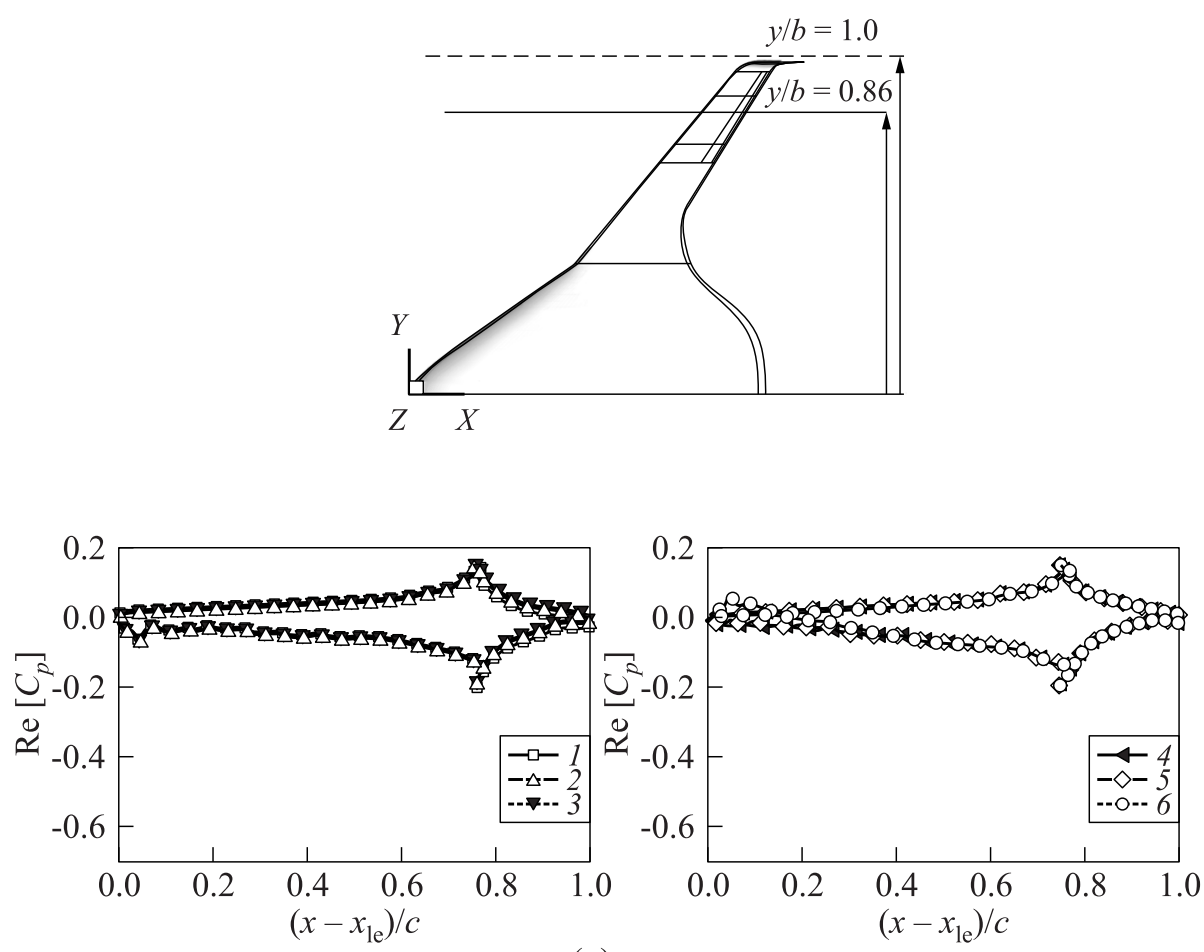

(a)

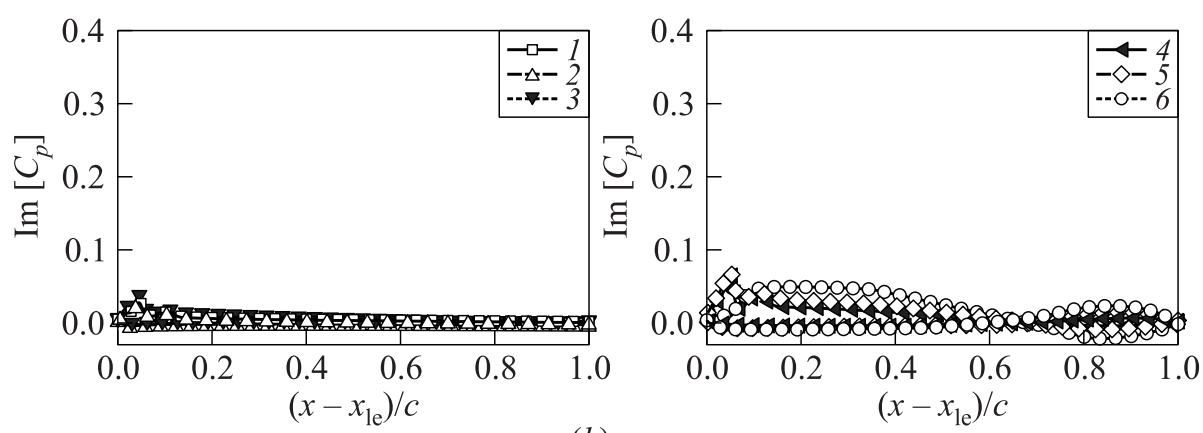

(b)

Figure 5 Real $(a)$ and imaginary $(b)$ parts of the pressure coefficient for oscillating Flap5, $\mathrm{M}=0.75 ; y / b=0.86: 1-k_{\mathrm{red}}=0.01 ; 2-0.05 ; 3-0.1 ; 4-0.5 ; 5-1.0$, and $6-k_{\text {red }}=2.0$ 

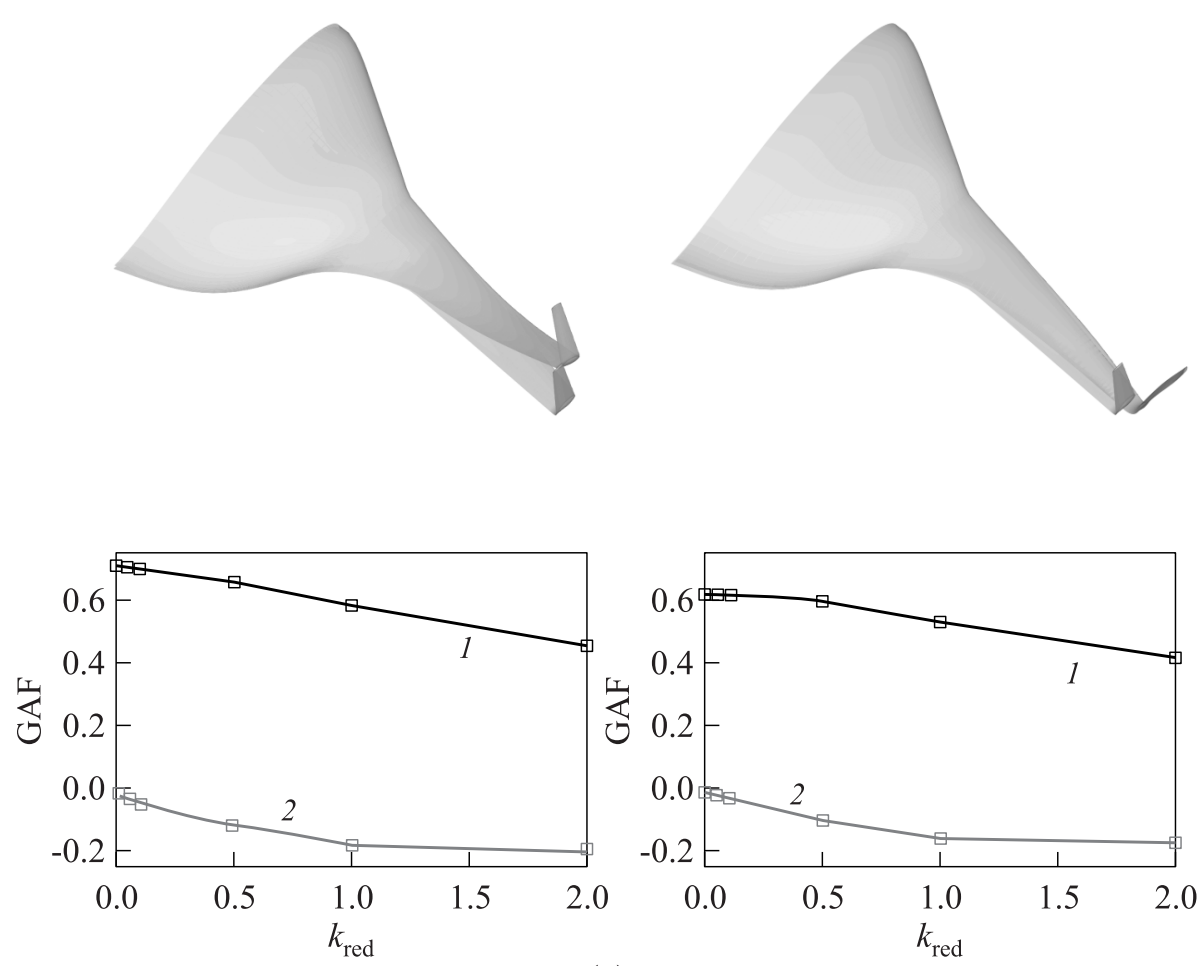

(a)
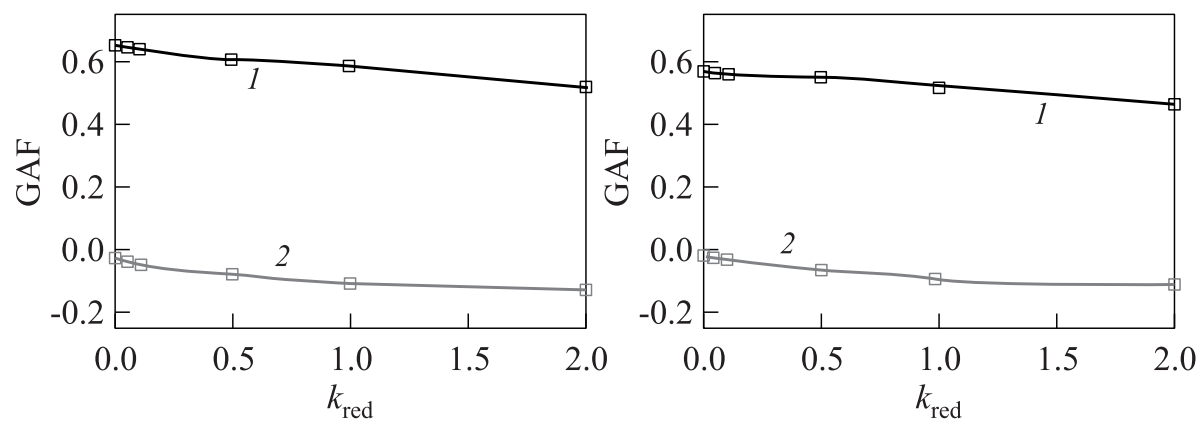

(b)

Figure 6 Real (1) and imaginary (2) parts of GAF at $\mathrm{M}=0.85(a)$ and $\mathrm{M}=0.75(b)$ for oscillating Flap5 projected on first bending (left column) and first torsion (right column) 
The real part of $C_{p}$ is only slightly affected by the oscillation at the three lower frequencies. Similar to the steady results, a shock is located at $\left(x-x_{\mathrm{le}}\right) / c \approx 0.6$ for $\mathrm{M}=0.85$ as opposed to $\mathrm{M}=0.75$ (see the plots for $y / b=0.86$ in left columns of Figs. 4 and 5).

Instead, for $\mathrm{M}=0.75$, a recompression zone is located at $\left(x-x_{\mathrm{le}}\right) / c \approx 0.1$ similar to the steady results. For both flight speeds, a second discontinuity is located at the leading edge of Flap5 (at $\left.\left(x-x_{\mathrm{le}}\right) / c=0.75\right)$. It is flight speed independent and is due to the flap oscillation. A further frequency increase mitigates the real part of the shock pressure peak for $\mathrm{M}=0.85$ and the recompression for $\mathrm{M}=0.75$ while the discontinuity due to the flap oscillation remains unchanged.

On the imaginary part of $C_{p}$, a frequency increase also has an affect at the lower frequencies. The intensity of the shock increases and, therefore, implies a growing unsteadiness. Interestingly, the highest peak value is obtained for $k_{\text {red }}=1.0$ and not for the maximum value $k_{\text {red }}=2.0$. The recompression zone for $\mathrm{M}=0.75$ at $\left(x-x_{\mathrm{le}}\right) / c \approx 0.1$ grows and moves downstream. As opposed to the real part of $C_{p}$, in the imaginary part of $C_{p}$, no discontinuity is visible at the leading edge of Flap5. This implies that the discontinuity is steady and, therefore, frequency-independent.

The GAF coefficients for the oscillation of the Flap5 projected on the deformation due to the elastic modes, first (symmetric) bending and first (symmetric) torsion are shown in Fig. 6. For both bending and torsion movements, an increasing reduced frequency leads to a decrease of the steady part of the GAF and an increase in the unsteady part of the GAF which has a negative sign. No significant difference is visible between the results of the two flight Mach numbers.

Figures 7 and 8 show the real and the imaginary part of $C_{p}$ for the same mid-flap position for the case of an oscillating TED. With the same axis scaling as the figures for Flap5, no considerable influence is visible due to changes in the Mach number or reduced frequency of the oscillating TED.

Please note that for $\mathrm{M}=0.85$, similar to the steady results and the oscillating Flap5, a shock exists in the real part of $C_{p}$ at $\left(x-x_{\mathrm{le}}\right) / c=0.6$. For the higher three frequencies, the real part of the pressure is significantly mitigated.

However, with increasing reduced frequency, the shock unsteadiness grows. The highest peak is reached for $k_{\text {red }}=0.5$, i. e., the shock is highly unsteady for this frequency. Interestingly, for the two highest frequencies, the shock becomes "steady" again. For an oscillation at $k_{\text {red }}=2.0$, the shock is mitigated most.

Similarly, the effect on the elastic modes is significantly smaller for the TED than for the previously analyzed Flap5. No considerable influence of the reduced frequency is visible (Fig. 9). 

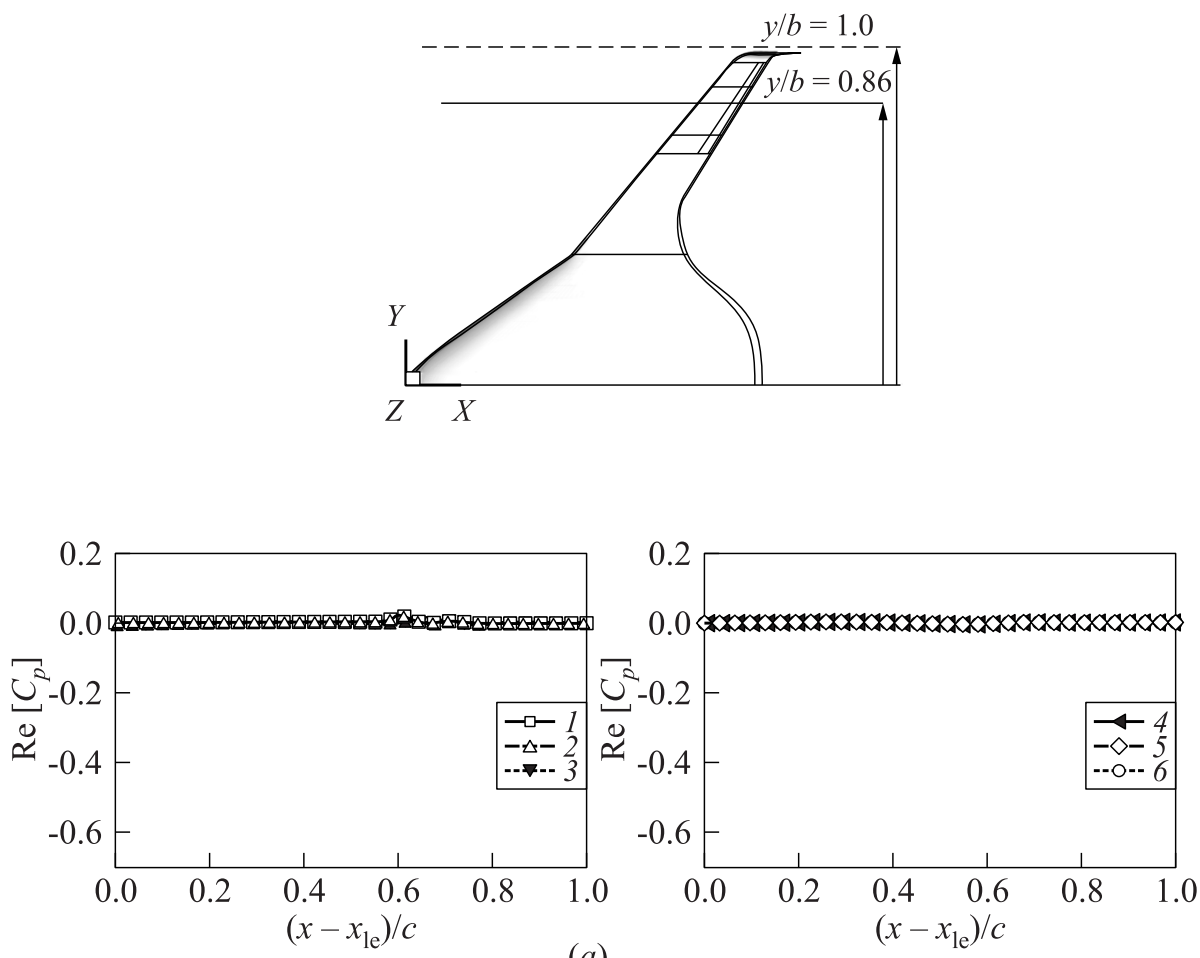

(a)
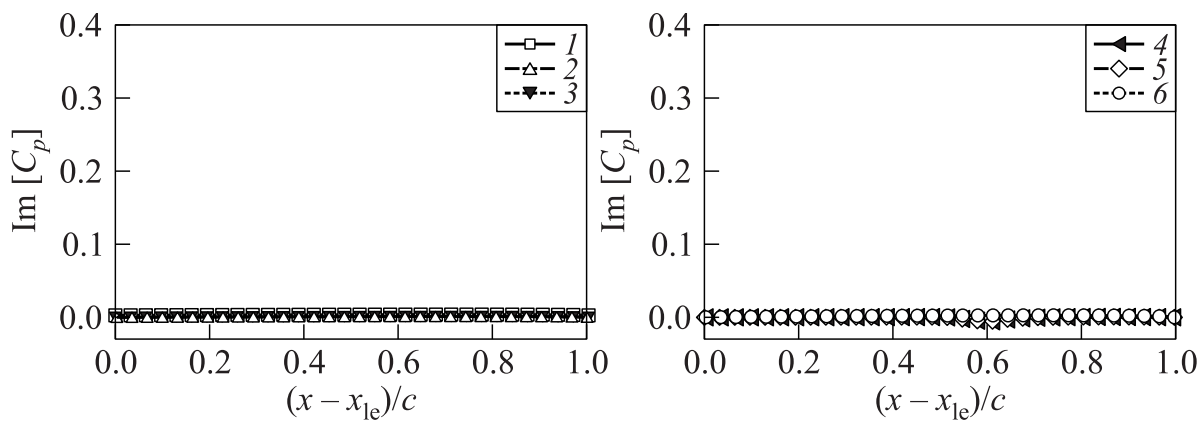

(b)

Figure 7 Real $(a)$ and imaginary $(b)$ parts of the pressure coefficient for oscillating TED; $\mathrm{M}=0.85 ; y / b=0.86: 1-k_{\mathrm{red}}=0.01 ; 2-0.05 ; 3-0.1 ; 4-0.5 ; 5-1.0$; and $6-k_{\text {red }}=2.0$ 

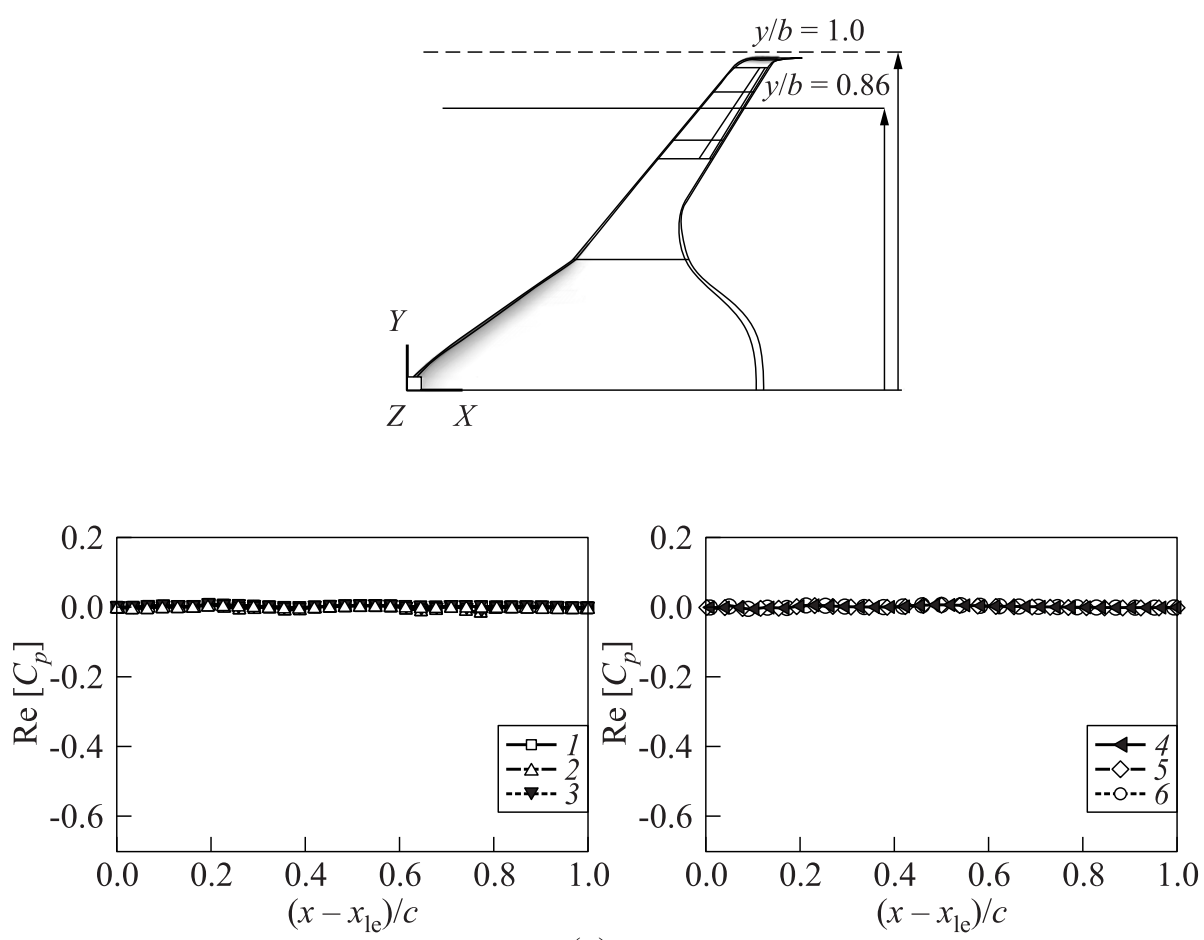

$(a)$
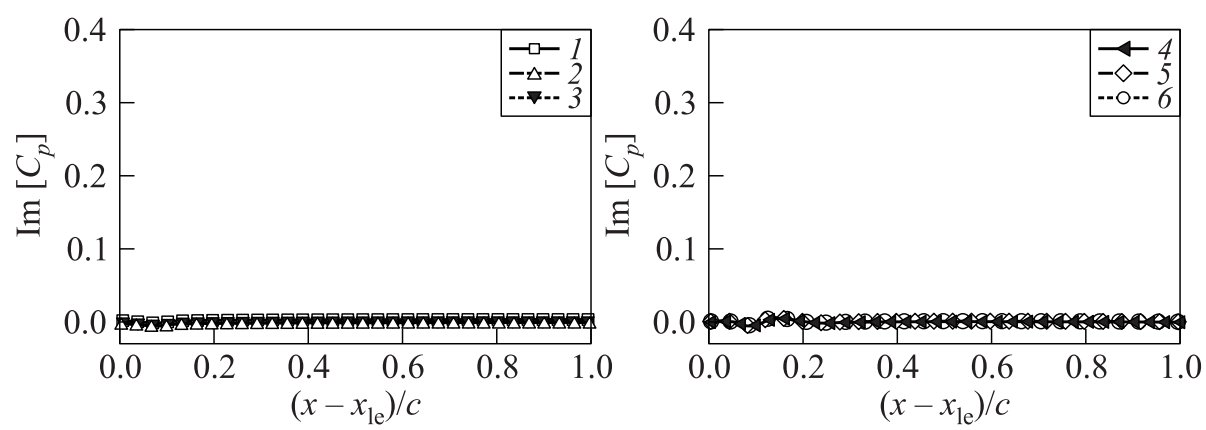

(b)

Figure 8 Real $(a)$ and imaginary $(b)$ parts of the pressure coefficient for oscillating $\mathrm{TED} ; \mathrm{M}=0.75 ; y / b=0.86: 1-k_{\mathrm{red}}=0.01 ; 2-0.05 ; 3-0.1 ; 4-0.5 ; 5-1.0$; and $6-k_{\text {red }}=2.0$ 

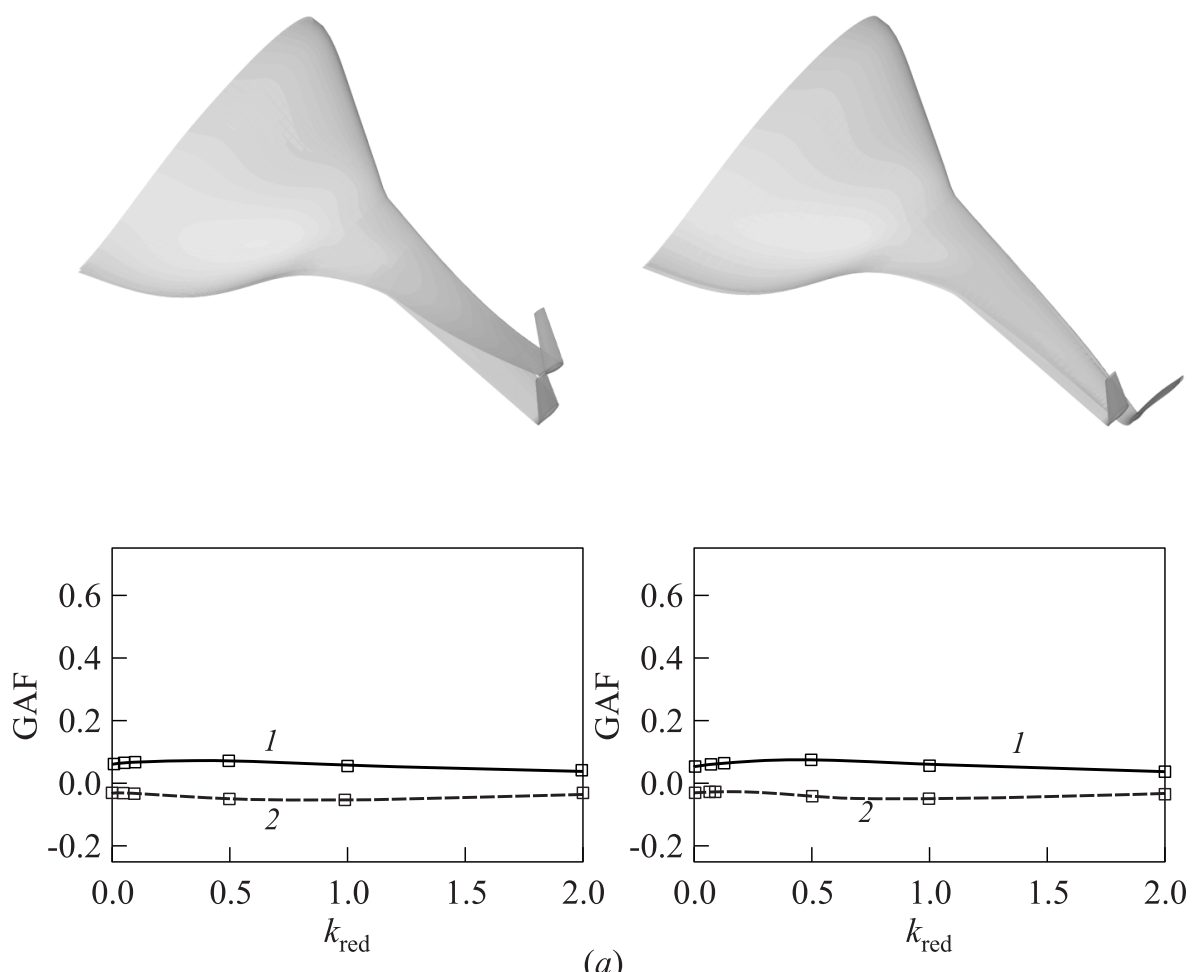

(a)
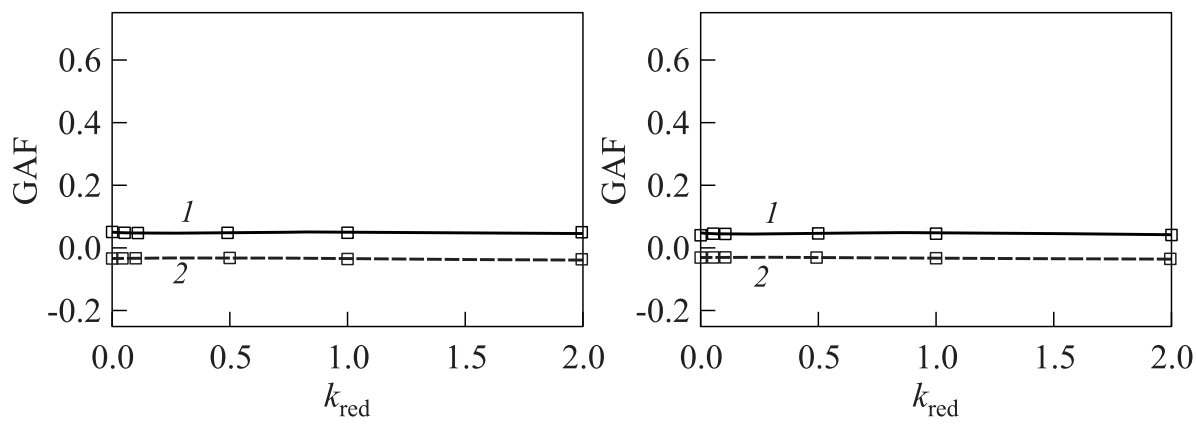

(b)

Figure 9 Real (1) and imaginary (2) parts of the GAF for oscillating TED projected on first bending (left column) and first torsion (right column) at $\mathrm{M}=0.85(a)$ and $0.75(b)$ 


\section{SUMMARY AND OUTLOOK}

In this work, the aerodynamics of the ACFA2020 BWB have been analyzed based on a limited number of test cases with time-accurate high-fidelity Euler simulations.

The steady results show a strong Mach number influence on the pressure distribution, i.e., the lift distribution. This influence is particularly severe on the outboard wing and leads to strongly varying shock positions.

The unsteady results for an oscillating Flap5/aileron at cruise conditions show that with increasing oscillation frequency, the shock on the outboard wing becomes unsteady but does not considerably change in intensity.

At a lower flight speed, the changes in reduced frequency are less significant. With increasing oscillation frequency, an existent recompression zone is stretched in streamwise direction and becomes unsteady. For both flight speeds, the oscillation of Flap5 at increasing frequency reduced the steady part of the unsteady forces and increased the unsteady part.

The unsteady results for an oscillating TED do not show any great effect of neither Mach number nor frequency compared to the oscillating Flap5. It can be concluded that the use of an oscillating aileron/Flap5 will be needed for active structural control.

\section{REFERENCES}

1. ACFA 2020 - Active Control for Flexible 2020 Aircraft: Available via DIALOG. http://www.acfa2020.eu/.

2. Mohr, B., D. Paulus, H. Baier, and M. Hornung. 2011. Design of a 450 passenger blended wing body aircraft for active control investigations. J. Aerospace. Eng. doi:10.1177.

3. Fleischer, D., C. Vidy, M. Iatrou, C. Breitsamter, and C. Weishäuptl. 2009. Linear flutter prediction for low aspect raio wings using a small disturbance Euler method. IFASD Forum (International) for Aeroelasticity and Structural Dynamics Proceedings. IFASD-2009-055.

4. Kreiselmaier, E., and B. Laschka. 2000. Small disturbance euler equations efficient and accurate tool for unsteady load prediction. J. Aircraft 37(5):770-78.

5. Harten, A. 1983. High resolution schemes for hyperbolic conservation laws. J. Comput. Phys. 49:357-93. 
6. Roe, P. L. 1981. Approximate Riemann solvers, parameter vectors and difference schemes. J. Comput. Phys. 43:357-72.

7. Blazek, J. 1993. Investigations of the implicit LU-SSOR scheme. Report DLR-FB 9351. Braunschweig, Germany: Institute for Design Aerodynamics, German Aerospace Research Center (DLR). 